\title{
Impacto da reabilitação com próteses fixas metal free na qualidade de vida
}

\author{
Impact of rehabilitation with fixed metal free prostheses \\ on the quality of life \\ Impacto de la rehabilitación com prótesis fijas metal free \\ en la calidad de vida \\ Adriana Cristina ZAVANELLI ${ }^{1}$ \\ Otavio Marino dos SANTOS NETO ${ }^{2}$ \\ José Victor Quinelli MAZARO ${ }^{1}$ \\ Adhara Smith NÓBREGA ${ }^{3}$ \\ Ricardo Alexandre ZAVANELLI ${ }^{4}$ \\ ${ }^{I}$ Departamento de Materiais Odontológicos e Prótese, Faculdade de Odontologia de Araçatuba, UNESP, \\ Univ. Estadual Paulista, 16015-050 Araçatuba - SP, Brasil \\ ${ }^{2}$ Mestrando em Odontologia (Reabilitação Oral) pela Faculdade Odontologia de Ribeirão Preto, USP, \\ Univ. de São Paulo, 14090-904 Ribeirão Preto-SP, Brasil \\ ${ }^{3}$ Doutoranda em Odontologia (Prótese Dentária) pela Faculdade de Odontologia de Araçatuba, UNESP, \\ Univ. Estadual Paulista, 16015-050 Araçatuba - SP, Brasil \\ ${ }^{4}$ Departamento de Reabilitação Oral, Faculdade de Odontologia, UFG \\ Universidade Federal de Goiás, 74605-020 Goiânia - GO, Brasil
}

\section{Resumo}

Nos tratamentos reabilitadores que envolvem a estética, é fundamental considerar a expectativa e a satisfação do paciente com o tratamento realizado. Não preencher as necessidades estéticas solicitadas pelo paciente pode acarretar de prejuízos emocionais a não adesão ao tratamento. Esta pesquisa teve como objetivo avaliar a qualidade de vida de pacientes reabilitados com próteses metal free. Foram avaliados todos os pacientes que aceitaram participar da pesquisa e que receberam tratamento reabilitador estético. Os pacientes foram avaliados imediatamente antes do início do tratamento e 30 dias após a conclusão do caso por meio do questionário de qualidade de vida OHIP-14. Os dados obtidos foram submetidos à análise estatística. Os resultados obtidos mostram que houve uma redução significativa em todos os domínios do OHIP-14. Logo, as reabilitações estéticas quando levam em consideração os anseios do paciente e seguem um protocolo de tratamento adequado, alcançam o sucesso tanto para a saúde oral e geral, quanto para o conforto e expectativas do paciente.

Descritores: Reabilitação Bucal; Estética Dentária; Qualidade de Vida.

\begin{abstract}
In the rehabilitation treatments which involve the aesthetic is fundamental to considerate the expectative and the satisfaction of the patient with the treatment accomplished. Not to fill the aesthetic necessities requested by the patient can be into emotional damages and no-adherence to treatment. This investigation has the aim to evaluate quality of life of the patients rehabilitated with metal free prosthesis. Were evaluated all the patients that accepted to participate of the investigation and that received aesthetic rehabilitation treatment. The patients were evaluated before the start of the treatment and 30 days after the conclusion of the case by means of the questionnaire of the quality of life OHIP-14. The data obtained were submitted to statistical analysis. The results obtained show that there was an significant reduction in all the OHIP-14 domains. Presently, the aesthetic rehabilitations when take into account the wishes of the patient and follow an suitable treatment protocol, these achieve the success both for the oral health and general, as for comfort and expectations of the patient.

Descriptors: Mouth Rehabilitation; Esthetics, Dental; Quality of Life.
\end{abstract}

\section{Resumen}

En los tratamientos de rehabilitadores que envuelven la estética, es fundamental considerar la expectativa y la satisfacción del paciente con el tratamiento realizado. No llenar las necesidades estéticas solicitadas por el paciente puede acarrear en perjuicios emocionales a la no no adhesión al tratamiento. Esta investigación tuvo como objetivo evaluar la calidad de vida de pacientes rehabilitados con prótesis metal free. Fueron evaluados todos los pacientes que aceptaron participar de la investigación y que recibieron tratamiento rehabilitador estético. Se evaluaron todos los pacientes que aceptaron participar de la pesquisa y que recibieron tratamiento rehabilitador estético. Los pacientes fueron evaluados inmediatamente antes del inicio del tratamiento y 30 días después de la conclusión del caso a través del cuestionario de calidad de vida OHIP-14. Los dados obtenidos se sometieron al análisis estadístico. Los resultados muestran que hubo una reducción significativa en todos los dominios del OHIP-14. Por lo tanto, las rehabilitaciones estéticas cuando toman en consideración los anhelos del paciente y siguen un protocolo de tratamiento adecuado, alcanzan el éxito tanto para la salud oral y general, como para la confortación y expectativas del paciente.

Descriptores: Rehabilitación Bucal; Estética Dental; Calidad de Vida.

\section{INTRODUÇÃO}

A estética é o estudo racional do belo, quer quanto à possibilidade da sua conceituação, quer quanto à diversidade de emoções e sentimentos que ele sucinta no homem. A estética pode ainda ser definida como a filosofia das belas artes ou ciência que trata do belo, na natureza e na arte $^{1}$. Em outras palavras, é aquilo que reconhecemos como agradável sem perceber o porquê.

Embora existam parâmetros matemáticos de proporção, a beleza é única, isso torna a restauração do sorriso ou a reparação de danos causados pelo tempo não apenas um ato técnico. Além de evidências científicas é necessário dom artístico aliado ao bom senso para estabelecer proporções e criar harmonia ${ }^{2}$.

$\mathrm{O}$ rosto representa muitos sentimentos e emoções do ser humano sendo o sorriso uma das estruturas faciais que mais transmitem informações, pois no sorriso movimentamos muitos músculos da face, expondo dessa maneira dentes e gengiva, movimentamos lábios, face e até o olhar ${ }^{3}$. Um sorriso agradável pode produzir uma aura que amplia a beleza da face, fazendo parte das qualidades e 
virtudes da personalidade; pode, ainda, exprimir emoções negativas e ideias, como agrado, desagrado, medo e miséria $^{4-5}$. Assim, o seu comprometimento pode levar as pessoas a recorrerem a meios sutis ou explícitos de esconder a boca, o que afeta aspectos do estilo de vida. Pessoas com problemas estéticos dentários têm baixa autoestima e tendem a cobrir a boca quando falam, movendo, às vezes, os lábios de forma artificial ${ }^{5-6}$.

Nos tratamentos reabilitadores que envolvem a estética, é fundamental considerar a expectativa e a satisfação do paciente com o tratamento realizado. Não preencher as necessidades estéticas solicitadas pelo paciente pode acarretar prejuízos emocionais afetando sua autoestima e relacionamentos interpessoais ${ }^{7}$.

A avaliação do impacto dos tratamentos estéticos na qualidade de vida dos pacientes é uma temática que tem aumentado nos últimos anos ${ }^{8}$. Qualidade de vida (QV) enfoca o aspecto humano da existência. No campo da saúde, esta temática reflete individual e coletivamente as dimensões subjetividade, multidimensionalidade e bipolaridade na ocorrência ou tratamento de doenças e traumas?.

A Organização Mundial da Saúde (OMS) define QV como "a percepção do indivíduo de sua posição na vida, no contexto da cultura e sistemas de valores nos quais vive e em relação aos seus objetivos, expectativas, padrões e preocupações" 10

A utilização de instrumentos de medida para determinar a percepção do indivíduo, após o tratamento, é um reflexo verdadeiro da opinião do paciente, quanto às suas expectativas, frustrações ou satisfação. O questionário Perfil de Impacto de Saúde (Oral Health Impact Profile OHIP-14) desenvolvido por Slade e Spencer (1994) originalmente possui 49 questões e na sua versão abreviada 14 , é o instrumento mais eficaz, já desenvolvido para avaliar as mudanças na qualidade de vida decorrentes de alterações na saúde bucal ${ }^{11-14}$.

Diante do exposto, este trabalho teve como objetivo avaliar a qualidade de vida dos pacientes que receberão tratamento odontológico estético.

\section{MATERIAL E MÉTODO}

Esse estudo foi submetido ao Comitê de Ética em Pesquisa, sendo aprovado sob número CAAE: 44799115.2.0000.5420. Foram selecionados pacientes que necessitam de tratamento com prótese parcial fixa, atendidos na clínica de Prótese Parcial Fixa da Faculdade de Odontologia de Araçatuba - UNESP. Foi utilizado como critério de inclusão, pacientes submetidos a tratamento odontológico onde foram realizadas próteses parciais fixas convencionais do tipo metal free e que tinham capacidade cognitiva suficiente para responder aos questionários. Foram excluídos pacientes que se recusaram a preencher os questionários, menores de 18 anos de idade e pacientes com próteses metálicas, metalocerâmicas ou de resina composta laboratorial. Dentro dos critérios de inclusão e exclusão foram selecionados 35 pacientes.

Nesta pesquisa foi utilizado um instrumento para a avaliação da qualidade de vida, o Oral Health Impact Profile-14 (OHIP-14). As avaliações foram realizadas antes do início do tratamento reabilitador estético e 30 dias após a instalação das próteses. Os questionários foram respondidos pelos pacientes na sala de espera e em seguida foi realizada a assinatura do Termo de Consentimento Livre e Esclarecido foi preenchido em duas vias e após as assinaturas, uma via foi entregue ao paciente.
Os dados obtidos foram tabulados e analisados no Microsoft Office Excel 2013. Foi utilizada a escala de Likert para pontuar as respostas de cada pergunta do OHIP14 com os seguintes valores: $0=$ nunca, $1=$ raramente, $2=$ às vezes, $3=$ com frequência, $4=$ sempre, com pontuação máxima possível chegando a 56 pontos. Foram respeitados os sete domínios do OHIP, que classificam as perguntas de acordo com o que está sendo avaliado (tabela 1). O impacto geral é dado pela soma do impacto dos domínios e foi considerado fraco com índice menor que 9,33, médio com índice entre 9,33 e 18,66 , e forte com índice maior que 18,66 .

Tabela 1. Domínios do OHIP-14

\begin{tabular}{|c|c|}
\hline & \\
\hline Pergunta do questionário & Domínio \\
\hline $\begin{array}{l}\text { 1. Você teve problemas para falar } \\
\text { alguma palavra? }\end{array}$ & 1. Limitação \\
\hline $\begin{array}{l}\text { 2. Você sentiu que o sabor dos } \\
\text { alimentos tem piorado? }\end{array}$ & funcional \\
\hline $\begin{array}{l}\text { 3. Você sentiu dores na sua boca ou } \\
\text { nos seus dentes? }\end{array}$ & \\
\hline $\begin{array}{l}\text { 4. Você se sentiu incomodado ao } \\
\text { comer algum alimento? }\end{array}$ & 2. Dor física \\
\hline 5. Você ficou preocupado? & 3. Desconforto \\
\hline 6. Você se sentiu estressado? & psicológico \\
\hline $\begin{array}{l}\text { 7. Sua alimentação ficou } \\
\text { prejudicada? }\end{array}$ & 4. Incapacidade \\
\hline $\begin{array}{l}\text { 8. Você teve que parar suas } \\
\text { refeições? }\end{array}$ & física \\
\hline $\begin{array}{l}\text { 9. Você encontrou dificuldade para } \\
\text { relaxar? }\end{array}$ & 5. Incapacidade \\
\hline 10. Você se sentiu envergonhado? & psicorogica \\
\hline $\begin{array}{l}\text { 11. Você ficou irritado com outras } \\
\text { pessoas? }\end{array}$ & 6. Incapacidade \\
\hline $\begin{array}{l}\text { 12. Você teve dificuldades para } \\
\text { realizar suas atividades diárias? }\end{array}$ & social \\
\hline $\begin{array}{l}\text { 13. Você sentiu que sua vida, em } \\
\text { geral, ficou pior? }\end{array}$ & 7 Dofi. \\
\hline $\begin{array}{l}\text { 14. Você ficou totalmente incapaz de } \\
\text { realizar suas tarefas diárias? }\end{array}$ & 1. Deficiencia \\
\hline
\end{tabular}

Os escores encontrados no OHIP-14 foram tabulados e foi realizado o teste estatístico $\mathrm{T}$ de Student pareado, ao nível de 5\% de significância. Os resultados de cada resposta dada pelos pacientes foram discriminados e analisados suas respectivas médias e desvio padrão antes e depois. Por meio de valores absolutos - escore de qualidade de vida - foram comparadas as variações entre a amostra antes e depois da instalação da prótese fixa.

\section{RESULTADOS}

Com relação aos dados segundo a escala de Likert, foi possível estabelecer a média dos valores encontrados antes e depois do tratamento previsto (Tabela 2).

De acordo com as médias dos valores encontrados, os dados refletem para um impacto médio $(12,14)$ encontrado anteriormente a instalação das próteses parciais fixas metal free que obteve uma redução significativa $(4,22)$ posteriormente a instalação, indicando um impacto baixo.

Os escores encontrados no OHIP-14 foram tabulados e foi realizado o teste estatístico T de Student pareado, ao nível de 5\% de significância (Tabela 3).

$\mathrm{Na}$ análise dos valores médios e correspondentes ao desvio padrão, verificou-se que houve diferença estatística significante na comparação do impacto da saúde bucal na qualidade de vida dos pacientes antes e após da instalação das próteses parciais fixas metal free $(\mathrm{p}<0,05)$ (Tabela 3$)$. 
Tabela 2. Média dos valores encontrados de acordo com os domínios de OHIP-14

\begin{tabular}{l|c|c|c}
\hline \multirow{2}{*}{$\begin{array}{c}\text { OHIP - Domínios e } \\
\text { Total }\end{array}$} & \multirow{2}{*}{ Alcance } & \multicolumn{2}{c}{ Tempo } \\
\cline { 3 - 4 } & & Antes & Depois \\
\hline Limitação funcional & $0-8$ & 0,63 & 0,17 \\
\hline Dor física & $0-8$ & 2,31 & 1,08 \\
\hline $\begin{array}{l}\text { Desconforto } \\
\text { psicológico }\end{array}$ & $0-8$ & 3 & 1,28 \\
\hline Incapacidade física & $0-8$ & 1,37 & 0,54 \\
\hline $\begin{array}{l}\text { Incapacidade } \\
\text { psicológica }\end{array}$ & $0-8$ & 2,46 & 0,54 \\
\hline Incapacidade social & $0-8$ & 1,6 & 0,48 \\
\hline Deficiência & $0-8$ & 0,77 & 0,11 \\
\hline Total & $0-56$ & 12,14 & 4,22 \\
\hline
\end{tabular}

Tabela 3. Valores médios e correspondentes desvios padrão, do resultado final de qualidade de vida

\begin{tabular}{l|c|c|c|c}
\hline \multirow{3}{*}{ OHIP } & Tempo & Média & Desvio-padrão & p-valor \\
\cline { 2 - 4 } & Antes & 0,86 & 0,47 & \multirow{2}{*}{0,0006} \\
\cline { 2 - 4 } & Depois & 0,30 & 0,22 & \\
\hline
\end{tabular}

\section{DISCUSSÃO}

Na Odontologia, ainda é pouco difundida a ideia de qualidade de vida. A utilização de questionários validados propicia suporte científico, por meio de dados estatísticos, para mensurar o bem-estar físico, emocional e psíquico, denominados Qualidade de Vida ${ }^{15}$. O questionário OHIP-14 tem sido aplicado em diversos estudos em Odontologia, o que o consagra como instrumento fidedigno para a utilização em pesquisas que envolvam qualidade de vida.

Os tratamentos protéticos apresentam uma gama de implicações bastante específicas. A prótese parcial fixa é frequentemente de custo elevado, muitas vezes de longa duração, e seu sucesso depende de acurado julgamento clínico, da habilidade nos procedimentos clínicos e laboratoriais, e da expectativa do paciente. As falhas decorrem tanto das dificuldades técnicas quanto da não sintonia entre as expectativas do paciente, a percepção das características das restaurações, a análise do profissional e o objetivo do tratamento ${ }^{16}$. Os pacientes possuem cada vez mais expectativas em relação a um tratamento sofisticado para substituir os dentes ausentes, sendo que os mesmos estão cobrando e participando ativamente na tomadas de decisões durante o tratamento ${ }^{17}$.

Nos últimos anos observou-se um aumento na qualidade e quantidade de restaurações fabricadas em cerâmica pura. Este aumento deve-se principalmente à superioridade estética que estas restaurações apresentam comparativamente às restaurações metalocerâmica ${ }^{18}$. Apesar do sucesso alcançado pelas restaurações metalocerâmicas, a Odontologia sempre buscou a substituição do metal, principalmente por motivos estéticos. Mas, somente após o surgimento dos procedimentos adesivos e das cerâmicas reforçadas metal free, foi possível o restabelecimento estético com segurança e qualidade ${ }^{19,20}$

Este estudo apesar de suas limitações mostrou que o OHIP-14 foi eficiente para ilustrar o impacto na qualidade de vida dos pacientes reabilitados com prótese parcial fixa metal free. Os resultados mostram claramente que ocorreu redução significativa em todos os domínios do OHIP-14. Entretanto, as reduções mais significativas estão com relação aos aspectos psicológicos (desconforto e incapacidade psicológica), sugerindo que os pacientes avaliados de alguma maneira sentiram-se mais confiantes com o tratamento reabilitador estético.
Sendo assim, a utilização de instrumentos de medida validados, para determinar a percepção do indivíduo, após o tratamento, é reflexo verdadeiro da opinião do paciente, quanto às suas expectativas, frustrações ou satisfações, independente da interferência dos procedimentos clínica ${ }^{21}$. A intuição indica uma relação muito intensa entre vida e saúde, inclusive, há inúmeras evidências da contribuição da saúde para a qualidade de vida e vice-versa, alertando sobre a importância da promoção da saúde ${ }^{23}$.

\section{CONCLUSÃO}

As reabilitações estéticas quando levam em consideração os anseios do paciente e seguem um protocolo de tratamento adequado alcançam o sucesso tanto para a saúde oral e geral quanto para o conforto e expectativas, tendo como pilar mestre a opinião do paciente.

\section{REFERÊNCIAS}

1. Borghetti A, Monnet-Corti V. Cirurgia plástica periodontal. Porto Alegre: Artmed; 2002.

2. Joly JC, Mesquita PFC, Silva RC. Princípios cirúrgicos em plásticas periodontal e perimplantar. In: Reconstrução tecidual estética: procedimentos plásticos e regenerativos periodontais e peri-implantares. São Paulo: Artes Médicas; 2010. p.153-88.

3. Farias BC, Gusmão ES, Fernandes AV, Valença ASM, Moreira MF, Cimões R. Determinação dos tipos de sorriso. Int J Dent. 2007; 6(3):80-3.

4. Qualtrough AJ, Burke FJ. A look at dental esthetics. Quintessence Int. 1994; 25(1):7-14.

5. Lichter JA, Solomowitz BH, Sauco M, Sher M. What's behind your smile? NY State Dent J. 1999; 65(10):34-9.

6. Hungerford M. Conceitos de estética dental - A beleza está nos olhos de quem vê. In: Goldstein RE. A estética em odontologia. 2. ed. São Paulo: Santos; 2000.

7. Ferreira AAA, Piuvezam G, Werner CWA, Alves MSCF. A dor e a perda dentária: representações sociais do cuidado à saúde bucal. Ciênc saúde coletiva. 2006; 11(1):211-8

8. Silva GR, Roscoe MG, Ribeiro CP, Mota AS, Martins LRM, Soares CJ. Impact of rehabilitation with metalceramic restorations on Oral Health-Related Quality of Life. Braz Dent J. 2012; 23(4):403-8.

9. Dantas RAS, Sawada NO, Malerbo MB. Pesquisas sobre qualidade de vida: revisão da produção científica das universidades públicas do Estado de São Paulo. Rev Latino-Am Enfermagem. 2003; 11(4):532-8.

10. The World Health Organization Quality of Life assessment (WHOQOL): position paper from the World Health Organization. Soc Sci Med. 1995; 41(10):1403-9.

11. Cohen-Carneiro F, Rebelo MA, Souza-Santos R, Ambrosano GM, Salino AV, Pontes DG. Psychometric properties of the OHIP-14 and prevalence and severity of oral health impacts in a rural riverine population in Amazonas State, Brazil. Cad Saúde Pública. 2010; 26(6):1122-30.

12. Oliveira BH, Nadanovsky P. Psychometric properties of the Brazilian version of the Oral Health Impact Profile short form. Community Dent Oral Epidemiol. 2005; 33(4):307-14.

13. Pires CP, Ferraz MB, De Abreu MH. Translation into Brazilian Portuguese, cultural adaptation and validation of the oral health impact profile (OHIP-49). Braz Oral Res. 2006; 20(3):263-8.

14. Slade GD. Derivation and validation of a short form of Oral Heath Impact Profile. Comm. Dent. Health. 1997; 25:284-290. 
15. Weintraub JA. Uses of oral health related quality of life measures in public health. Community Dent Health. $1998 ; 15(1): 8-12$

16. Albino JE, Tedesco LA, Conny D. Patitent perceptions of dental-facial esthetics: shared concerns in orthodontics and prosthodontics. J Prosthet Dent.1984; 52(1):9-13.

17. Cronin M, Meaney S, Jepson NJ, Allen PF. A qualitative study of trends in patient preferences for the management of the partially dentate state. Gerodontology. 2009; 26(2):137-42.

18. Gordilho AC, Mori M, Gil C, Contin I. A adaptação marginal dos principais sistemas de cerâmica pura, Revista Odonto. 2009; 17(34):82-92.

19. Bowen RL. Properties of a sílica-reinforced polymer for dental restorations. J Am Dent Assoc 1963; 66:57-64.

20. Buonocore MG. A simple method of increasing the adhe-sion of acrylic filling materials to enamel surfaces. J Dent Res. 1955; 34(6):849-53.

21. Allen PF, Locker D. Do item weights matter? An assessment using the oral health impact profile. Community Dent Health. 1997; 14(3):133-8.

22. Ferraz MB. Qualidade de vida: conceito e um breve histórico. Rev Jovem Médico. 1998; 4:219-22.

\section{CONFLITO DE INTERESSES}

Os autores declaram não haver conflitos de interesse.

\section{AUTOR PARA CORRESPONDÊNCIA}

\section{Adriana Cristina Zavanelli}

zavanelliac@foa.unesp.br

Submetido em 12/07/2017 Aceito em 25/08/2017 